\title{
Evidence for Holistic Representations of Ignored Images and Analytic Representations of Attended Images
}

\author{
Volker Thoma \\ University of London
}

\author{
John E. Hummel \\ University of California, Los Angeles
}

\author{
Jules Davidoff \\ University of London
}

\begin{abstract}
According to the hybrid theory of object recognition (J. E. Hummel, 2001), ignored object images are represented holistically, and attended images are represented both holistically and analytically. This account correctly predicts patterns of visual priming as a function of translation, scale (B. J. Stankiewicz \& J. E. Hummel, 2002), and left-right reflection (B. J. Stankiewicz, J. E. Hummel, \& E. E. Cooper, 1998). The model also predicts that priming for attended images will generalize over configural distortions (split images), whereas priming for ignored images will not. Three experiments tested and confirmed this prediction. Split images visually primed their intact and split counterparts when they were attended but not when they were ignored, whereas intact images primed themselves whether they were attended or not. The data contribute to the growing body of evidence that 1 function of visual attention is to permit the generation of explicitly relational representations of object shape.
\end{abstract}

The human capacity for visual object recognition is characterized by a number of properties that are jointly very challenging to explain. Among the most notable is that the visual representation of shape is invariant with (i.e., insensitive to) the location of the image in the visual field (Biederman \& Cooper, 1991a), the size of the image (Biederman \& Cooper, 1992), left-right (i.e., mirror) reflection (Biederman \& Cooper, 1991a; Davidoff \& Warrington, 2001), and some rotations in depth (Biederman \& Gerhardstein, 1993, 1995; but see Tarr \& Bülthoff, 1995). Object recognition is also remarkably robust to variations in shape (Davidoff \& Warrington, 1999). For example, a child's drawing of a car may be easily recognizable as a car, even if it resembles neither any particular real car nor any road-worthy object. At the same time, object recognition is sensitive to rotations about the line of sight (Jolicœur, 1985; Tarr \& Pinker, 1989, 1990) and some rotations in depth (see Lawson, 1999, for a review).

The combination of view-invariant and view-dependent properties of object recognition is problematic for theories that rely exclusively on the geometric properties of object shape-for example, by matching 2-D images to 3-D models in memory (e.g.,

Volker Thoma and Jules Davidoff, Department of Psychology, Goldsmiths College, University of London, London, United Kingdom; John E. Hummel, Department of Psychology, University of California, Los Angeles.

This research was supported by a German Academic Exchange Service fellowship awarded to Volker Thoma. Additional support was provided by a University of London Central Research Fund grant to Volker Thoma.

Correspondence concerning this article should be addressed to Volker Thoma, who is now at the Department of Psychology, University College London, University of London, 26 Bedford Way, London WC1H 0AP, United Kingdom. E-mail: v.thoma@ucl.ac.uk
Lowe, 1987; Ullman, 1989, 1996) or by using mathematical interpolation to determine whether a given image is a "legal" projection of a familiar shape (e.g., Poggio \& Edelman, 1990; Ullman \& Basri, 1991). A visual system that relied exclusively on the laws of projective geometry would be equally able to accommodate all variations in viewpoint (which the human visual system does not) but would not tolerate variations in object shape (which the human visual system does). Such a visual system would also fail to treat an image and its left-right reflection as equivalent.

In the attempt to better explain the properties of human object recognition, a number of researchers have proposed that objects are visually represented as structural descriptions specifying the object's features or parts in terms of their (typically categorical) interrelations (Biederman, 1987; Hummel \& Biederman, 1992; Hummel \& Stankiewicz, 1996b, 1998; Marr \& Nishihara, 1978; Palmer, 1977; Winston, 1975). For example (see Biederman, 1987), a coffee mug might be represented as a curved cylinder (the handle) side-attached to a straight vertical cylinder (the body). Like human shape perception, this description is unaffected by translation across the visual field, changes in size, left-right reflection, and some rotations in depth. However, it is sensitive to rotations about the line of sight (e.g., a $90^{\circ}$ rotation changes the side-attached relation between the cup handle and body to an above-attached relation). The description also applies to many different mugs, permitting generalization over metric variations in the shapes of a class of objects.

One of the most important properties of a structural description is that it is an analytic representation, meaning that it specifies the relations among an object's parts both explicitly and independently of the parts (Hummel, 2000; Hummel \& Biederman, 1992). For example, the spatial relation between the body and the handle of a coffee mug is explicitly described as "side-attached"; this spatial 
relation is independent of the parts it describes and may be used in conjunction with other parts to describe a different object. In contrast, a holistic representation, such as a 3-D model (e.g., Lowe, 1987; Ullman, 1989, 1996), a 2-D "view” (e.g., Poggio \& Edelman, 1990; Riesenhuber \& Poggio, 1999; Tarr \& Bülthoff, 1995), or an object "fragment" (Edelman \& Intrator, 2000, 2003), does not represent the relations among an object's parts explicitly or independently of those parts. Instead, an object's features or parts are represented in terms of their literal positions in a 2-D (in the case of view- and fragment-based models) or 3-D (in the case of model-based models) coordinate system (see Hummel, 2000, 2003a, for reviews).

Consistent with the structural description account of shape perception, there is evidence that the visual system represents the relations among an object's parts both explicitly (Hummel \& Stankiewicz, 1996b; Palmer, 1978; Tversky \& Hemenway, 1984) and independently of the parts themselves (Saiki \& Hummel, 1998a, 1998b). However, two properties of object perception are inconsistent with the properties of analytic representations. First, despite the substantial evidence that feature conjunctions require time-consuming attentional resources (Logan, 1994; Luck \& Vogel, 1997; Treisman \& Gelade, 1980), there is evidence that object recognition does not require visual attention. For example, numerous studies have demonstrated either negative priming (e.g., Murray, 1995; Tipper, 1985; Treisman \& DeSchepper, 1996) or positive priming for ignored object images (Stankiewicz \& Hummel, 2002; Stankiewicz et al., 1998). Whether the observed priming is positive or negative depends on whether the probe task requires the observer to actively ignore an irrelevant image. Second, both behavioral evidence (e.g., Intraub, 1981) and evidence from single-unit recording (e.g., Oram \& Perrett, 1992) indicate that object recognition is too fast to depend on analytic representations alone. Together, the speed and automaticity of object recognition suggest that structural descriptions cannot be the only basis for entry-level common object recognition (for a review, see Hummel, 2001).

In order to account for the speed and automaticity of common object recognition, while at the same time accounting for those properties of object recognition that are consistent with the role of structural descriptions, Hummel and Stankiewicz (1996a; Hummel, 2001) proposed a hybrid model of object recognition. The key idea behind the model is that holistic representations of shape can be generated rapidly and automatically because they do not represent object features or parts independently of their relations and, as a result, they do not require dynamic binding of parts to their relations. According to this model, attending to an object's image activates (and therefore visually primes) both a structural description of the object's shape and a holistic (i.e., view-like) representation of its shape; ignoring an image activates the holistic representation of its shape but not the structural description. The resulting model accounts for a large number of phenomena in human shape perception and object recognition (including all those cited above) and also generates several novel predictions (see Hummel, 2001).

One set of novel predictions concerns the relationship between visual attention and patterns of visual priming across left-right (i.e., mirror) reflections. The structural descriptions generated by the Hummel and Stankiewicz (1996a; Hummel, 2001) model are invariant with left-right reflection (i.e., the structural description the model generates in response to one image is identical to the structural description it generates in response to its left-right reflection; the same is true of the models of Hummel \& Biederman, 1992, and Hummel \& Stankiewicz, 1998). However, the model's holistic representations are sensitive to left-right reflection (i.e., an image and its reflection may generate nonoverlapping holistic representations). As such, the model predicts that attending to an object image will activate (and visually prime) both that image and its left-right reflection, whereas ignoring the image will activate and prime that image but not its reflection.

Stankiewicz et al. (1998) tested and confirmed the predictions of the hybrid analytic/holistic model in an object naming task with paired prime-probe trials. A prime trial consisted of a fixation cross followed by a cuing box to the left or right of fixation, followed by two line drawings of common objects, one appearing inside the cuing box and the other appearing on the other side of fixation. The participants' task was to name the cued image (the attended prime); they were not required to respond to the other image (the ignored prime). Each prime display was followed by a probe display containing a single image at fixation. The task was to name the object. The probe image depicted either the same object as the attended prime, the same object as the ignored prime, or an object the participant had not previously seen in the experiment (an unprimed probe, which served as a baseline). Probe images (except for unprimed probes) were either identical to the corresponding primes or were left-right reflections of them. Attended prime images reliably primed both themselves and their left-right reflections. However, ignored prime images only primed themselves. Moreover, the effects of attention (attended vs. ignored) and reflection (identical images vs. left-right reflections) were strictly additive: The priming advantage for same view prime-probe trials was equivalent in both attended and unattended conditions (about $50 \mathrm{~ms}$ ). The fact that attention and reflection had additive effects on priming provides strong support for the independence of the holistic and structured representations of shape in the hybrid model.

A second prediction of the Hummel and Stankiewicz (1996a; Hummel, 2001) hybrid model derives from the fact that the holistic representation, although sensitive to left-right reflection, is invariant with translation and scale. That is, even the representation generated on the holistic representation is independent of the object's location or size. The hybrid model predicts that priming for both attended and ignored images should be invariant with translation and scale. Stankiewicz and Hummel (2002) tested and confirmed this prediction. Although priming for attended images is globally greater than priming for ignored images, priming for both attended and ignored images is invariant with both translation and scale.

Although the findings of Stankiewicz and colleagues (Stankiewicz \& Hummel, 2002; Stankiewicz, Hummel, \& Cooper, 1998) are clearly consistent with the hybrid model, they do not provide direct support for the model's primary theoretical assertion, which is that object shape is represented in a hybrid analytic/holistic fashion. It is possible, for example, that both attended and ignored images are represented in a strictly holistic fashion but that attention permits the visual system to "flip" the representation of an image, permitting recognition (and priming) that is invariant over mirror reflections. Therefore, in the present experiments, we exploited the fact that analytic representations of shape are neces- 
sarily more robust to configural distortions (such as splitting an image down the middle and moving the left half of the image to the right-hand side; see Figure 1) than are holistic representations; this point bears elaboration.

Holistic representations are matched "all of a piece." For example, a holistic representation of the intact horse in Figure 1 (e.g., a view of the horse in a typical view-based model; e.g., Edelman, 1998; Poggio \& Edelman, 1990; Ullman \& Basri, 1991) would be matched, in its entirety, against an object's image to determine the degree of fit between the image and the holistic representation (i.e., view) in memory: The coordinates of the features in the viewed image would be matched to the coordinates of the features in the stored view, and the degree of fit would be computed as a function of the vector similarity of the coordinates of corresponding features. The process is directly analogous to laying a template for the intact horse over the image of the split horse and counting the points of overlap. By this holistic measure of similarity, the intact and split horse images are utterly different because no corresponding (and few, if any, similar) features reside in equivalent locations in the two images.

By contrast, to an analytic representation of shape, the intact and split images of the horse, although by no means identical, are nonetheless highly similar because they depict many of the same parts in many of the same spatial relations: Both depict a torso-like shape connected in various relations to leg- and head-like shapes. It is unimportant that the image-splitting procedure does not result in a "natural" parsing of the object into its parts. Splitting an image down the middle is very unlikely to parse it into pieces that correspond to part boundaries, but this is unproblematic for struc-
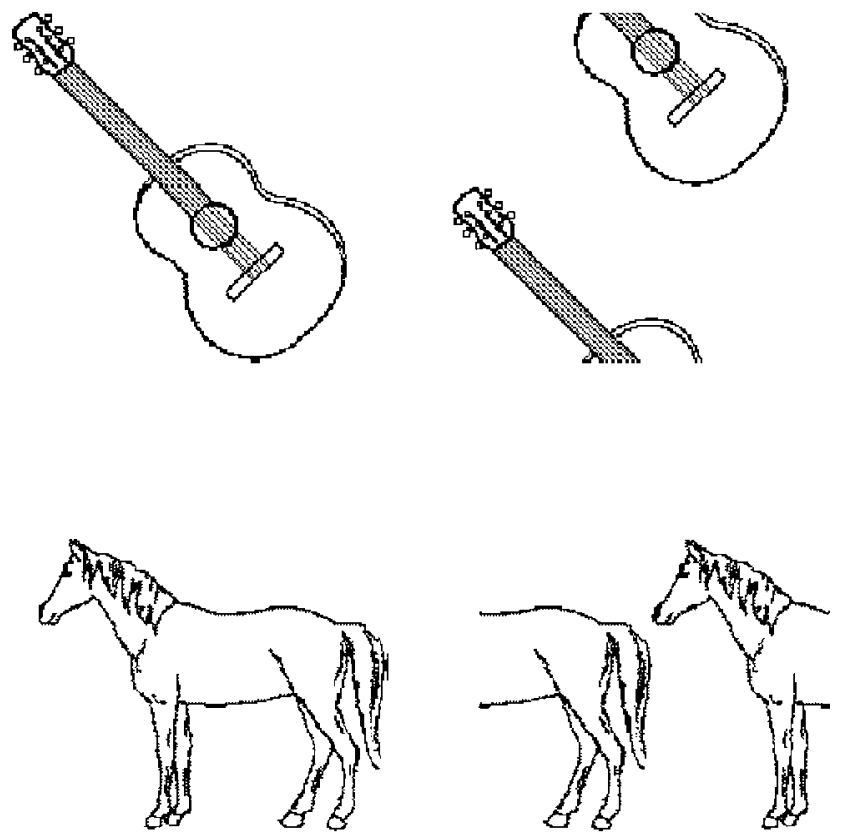

Figure 1. Examples of intact and split images used in Experiments 1-3. From "A Standardized Set of 260 Pictures: Norms for Name Agreement, Image Agreement, Familiarity, and Visual Complexity," by J. G. Snodgrass and M. Vanderwart, 1980, Journal of Experimental Psychology: Human Learning and Memory, 6, p. 200. Copyright 1980 by the American Psychological Association. Adapted with permission of the author. tural description theories as long as the shapes of the object's parts (such as the halves of the horse's torso in Figure 1) are recoverable from the information presented in each half of the image (Biederman, 1987; Hummel \& Biederman, 1992). And most if not all of the time, the parts' shapes will be recoverable (e.g., note that the horse is recognizable from either the front half or the left half alone). Recovery is easy because the relations between connected parts are much more important to shape perception than are relations between separated parts (Saiki \& Hummel, 1996, 1998a, 1998b). In the split image, the front of the horse is not connected to the back of the horse, so the resulting incorrect spatial relations between, say, the horse's head and its hind legs have little perceptual effect. Indeed, Cave and Kosslyn (1993) showed that split stimuli are substantially easier to recognize after short presentation times than are scrambled objects that disturb the spatial relationships between parts.

Some recent view-based models (notably Edelman \& Intrator, 2000, 2003) are based not on templates for whole objects but, rather, templates for object fragments - subsets of complete objects (e.g., the upper half of an object; see Edelman \& Intrator, 2003). Such models appear, at first blush, to be able to appreciate the similarity between a split image and its intact counterpart: A split image would seem to activate the same fragments as an intact version of the same image, thereby permitting visual priming from one to the other. However, even this fragment-based version of view-based theory predicts that a split image will not prime its intact counterpart. The reason is that the fragments these models use to represent object shape are tied to specific locations in the visual field (a representational scheme dubbed what + where coding; see Edelman \& Intrator, 2000, 2003). As a result, an intact object image will activate one set of fragments (e.g., a fragment for the front of a left-facing horse in the left part of the visual field and a fragment for the back half of the same horse in the right half of the visual field), and a split version of the same image will activate a completely separate set of fragments (e.g., a fragment for the front half of a left-facing horse in the right half of the visual field and a fragment for the back half of that horse in the left half of the visual field). Because the two sets of fragments are nonoverlapping, the model predicts no priming from one to the other (see Hummel, 2003b).

At the other extreme, Mel and colleagues (e.g., Mel, 1997; Mel \& Fiser, 2000) have proposed that the visual system recognizes objects simply by matching lists of location-invariant features to object memory. Such models are sensitive to which features are present in an object's image, but they are completely indifferent to the locations of those features (either relative to one another or in the image as a whole). These models predict that a split image will prime its intact counterpart (because the two depict many of the same features) but that this effect will obtain whether the split image is attended or not.

The general point is that without the ability to represent an object's parts independently of their locations, a model cannot predict priming from a split image to its intact counterpart. And without the ability to represent spatial relations explicitly (e.g., to represent which part is connected to which, located where relative to which, etc.), a model cannot separate an object's parts from their locations without losing the ability to represent how the parts are arranged. In other words, models based exclusively on holistic representations of shape - that is, conjunctions of shapes in par- 
ticular locations - cannot, in principle, predict that a split image could visually prime its intact counterpart. Only an analytic representation, such as a structural description - in which an object's parts are represented independently of their locations (and relations) - or a feature list (e.g., Mel, 1997), can predict that a split image will visually prime its intact counterpart. And of these models, only the hybrid model of Hummel and Stankiewicz (1996a; Hummel, 2001) predicts that the priming from a split image to its intact counterpart will be moderated by visual attention.

The present article reports three experiments designed to directly test the central theoretical assertion of the hybrid model, investigating whether the representation of an attended image is analytic and whether the representation of an ignored image is holistic. Experiment 1 investigated the role of attention in priming for split and intact object images. Participants named objects in pairs of prime-probe trials (as in Stankiewicz \& Hummel, 2002; Stankiewicz, et al., 1998). The first trial in a pair served as the prime and presented two object images, one of which (the attended image) was spatially precued. The participants' task was to name the precued object, ignoring the other (the ignored image). Half of the prime images were presented intact, and half were split either horizontally or vertically, as illustrated in Figure 1. The variables of attention (attended vs. ignored image) and image type (intact vs. split) were crossed orthogonally. The probe image was always intact and corresponded either to the attended prime, the ignored prime, or an image that the participant had not previously seen in the experiment (which served as a baseline). The hybrid analytic/ holistic model predicts that intact images will prime themselves whether they are attended or not (although priming should be greater for attended than for ignored images); split images should prime their intact counterparts when they are attended but not when they are ignored.

Experiment 2 served to estimate what fraction of the priming observed in Experiment 1 was specifically visual (as opposed to name or concept priming). Experiment 3 tested whether the priming for ignored images in Experiment 1 could be attributed to low-level visual representations (e.g., of the local features in an object's image) rather than holistic representations in long-term memory (LTM). On the former (low-level priming) account, ignored split images should prime themselves as much as ignored intact images prime themselves. On the latter (hybrid model) account, only ignored intact images should prime themselves.

\section{Experiment 1}

\section{Method}

Participants. Forty-two native English speakers with normal or corrected-to-normal vision participated for credit in introductory psychology courses at the University of California, Los Angeles.

Materials. Black-and-white line drawings of 84 asymmetrical objects from the Snodgrass and Vanderwart (1980) set were displayed on a PC monitor. Response times (RTs) were collected using E-Prime (Version 1.0) with a dynamic trigger microphone attached to an interface box. Participants sat approximately $90 \mathrm{~cm}$ from the display. The images were standardized in size to subtend $4.0^{\circ}$ of visual angle. For each object, a split version was created by using a $50 \%$ offset filter in Adobe Photoshop (Version 5.5), resulting in images that appeared to be cut into two halves that were relocated to the opposite side of the canvas (vertically or horizontally). The manipulation did not alter the total number of pixels in an image or the number or local configuration of any image features, except in that some lines were necessarily broken at the location of the cut (see Figure 1).

Procedure. The experimental conditions in which objects appeared were counterbalanced across participants by placing each image into one of 14 clusters, each containing six images, for the seven different primeprobe conditions. These conditions were attended-intact, attended-split, attended-not probed, ignored-intact, ignored-split, ignored-not probed, and unprimed. All primes preceded an intact probe image, and all images appeared in all seven conditions equally often across participants.

An image appeared in only 1 trial pair for any given participant. The ordering of the 36 trials and the pairing of attended and ignored objects on prime trials were randomized for each participant. The participants read instructions, which they then paraphrased back to the experimenter. The experimental session began with 18 practice trials using a set of images different from the experimental set. After the practice trials, the participants were asked whether they had any questions.

The sequence of events in a trial is depicted in Figure 2. An unfilled circle in the center of the screen remained until the participant started the trial by pressing the space bar. The circle was then replaced with a fixation cross, which remained on the screen for $495 \mathrm{~ms}$, followed by a blank white screen for $30 \mathrm{~ms}$. An attentional cuing square $4.5^{\circ}$ of visual angle on a side was then presented either to the left or to the right of the fixation cross, centered $4.0^{\circ}$ from fixation. After $75 \mathrm{~ms}$, two object images were displayed simultaneously for $120 \mathrm{~ms}$, with the attended image inside the square and the unattended image centered $4.0^{\circ}$ from fixation on the other side of the screen. The prime images could be both intact, both split or one of each. After the images disappeared, a blank screen was shown for $30 \mathrm{~ms}$,

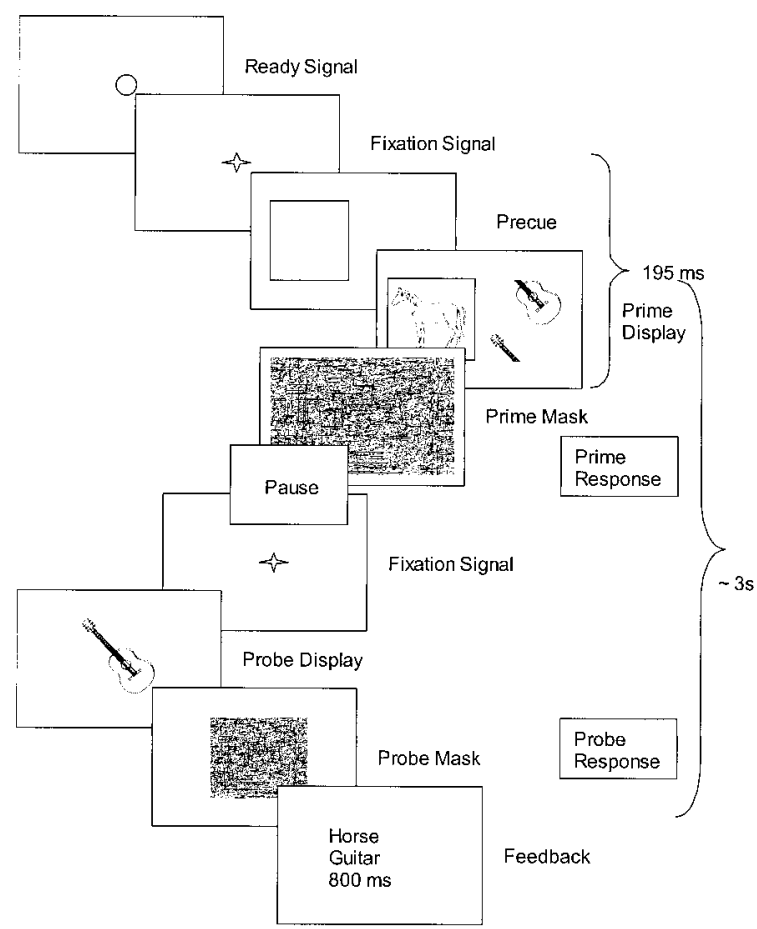

Figure 2. Sequence of displays in Experiment 1. Images are from "A Standardized Set of 260 Pictures: Norms for Name Agreement, Image Agreement, Familiarity, and Visual Complexity," by J. G. Snodgrass and M. Vanderwart, 1980, Journal of Experimental Psychology: Human Learning and Memory, 6, p. 200. Copyright 1980 by the American Psychological Association. Adapted with permission of the author. 
followed by a random-line pattern mask that covered the entire screen $\left(15.6^{\circ}\right.$ of visual angle) for $495 \mathrm{~ms}$. The entire prime display lasted less than $200 \mathrm{~ms}$, a duration too short to permit a saccade to the cuing square or either object. The participant's task at prime was to say the name of the cued (attended) object as quickly and as accurately as possible.

After the prime display, a blank screen was displayed for $1,995 \mathrm{~ms}$, followed by a fixation cross ( $495 \mathrm{~ms})$. Following a $30-\mathrm{ms}$ blank screen, the probe image was displayed in the center of the screen for $150 \mathrm{~ms}$. The probe depicted either the attended object (attended conditions), the ignored object (ignored conditions), or an object the participant had not seen previously in the experiment (unprimed baseline condition). The probe image was always intact. In total, 3,015 ms elapsed between the end of the prime display and the beginning of the probe display. The probe display was followed by a single pattern mask ( $4.6^{\circ}$ of visual angle) shown for 495 ms. The participant's task was to name the probe object as quickly and as accurately as possible. RTs at probe were recorded by the computer through a voice key attached to a microphone on the table.

The computer displayed the names of the attended prime and the probe object, as well as the probe RT. At the end of each trial pair, the experimenter used the keyboard to record the participant's accuracy as well as voice-key errors (i.e., when the voice key triggered erroneously). The participant could then initiate the next trial with a keypress.

\section{Results and Discussion}

In all conditions, priming was calculated as the participant's mean RT at probe in the unprimed (baseline) condition minus their mean RT in the corresponding experimental condition. Trials on which either the prime or probe responses were incorrect $(12.3 \%)$ were excluded from the statistical analysis, as were voice key errors $(3.9 \%)$. To verify that attention was deployed according to the instructions of each condition, the configuration (intact vs. split) of the unprobed prime was included as a variable in the analysis.

A 2 (attention: attended vs. ignored) $\times 2$ (prime configuration: intact vs. split) $\times 2$ (unprobed prime: intact vs. split) withinsubject analysis of variance (ANOVA) revealed reliable main effects of attention, $F(1,41)=138.77, p<.001$, and prime configuration, $F(1,41)=11.35, p<.002$, but the Attention $\times$ Prime Configuration interaction did not approach reliability, $F(1$,
41) $<1$ (see Figure 3 and Table 1). There was no main effect of unprobed prime or any interaction involving that factor (all $F_{\mathrm{S}}<$ 1). Thus, there was no indication of attention being deployed differently if both prime images were intact, split, or one of each. There were no indications of a speed-accuracy trade-off in any condition.

Analysis of each priming condition was carried out to determine which type of prime display caused savings in RT for the probe display (i.e., faster naming responses relative to unprimed probes). Priming was reliably greater than zero (Bonferroni corrected) in the attended-intact, $t(41)=13.85, p<.001$, attended-split, $t(41)=9.44, p<.001$, and ignored-intact conditions, $t(41)=$ $3.36, p<.01$, but not in the ignored-split condition, $t(41)<1$.

In general, the results of Experiment 1 are consistent with previous research, for both positive and negative priming, demonstrating that object recognition can take place in the absence of visual attention (e.g., Stankiewicz et al., 1998; Tipper \& Driver, 1988; Treisman \& DeSchepper, 1996). As predicted by the hybrid model, split images primed their intact counterparts only when they (the split images) were attended, but both attended and ignored intact images primed their intact counterparts. There was a reliable priming advantage for intact primes over split primes which was almost identical in both attended and ignored conditions ( $\sim 50 \mathrm{~ms}$, replicating Stankiewicz et al., 1998). Thus, the effects of attention (attended vs. ignored) and configuration (intact vs. split) were strictly additive. These results strongly support the hypothesis that two qualitatively different representations of shape mediate priming in object recognition: an analytic representation that is relatively robust to configural distortions but requires attention and a holistic representation that is sensitive to configural distortion but does not require attention.

\section{Experiment 2}

Experiment 1 necessarily confounded attention with naming: If and only if the participants attended an image did they name it. Therefore, it is at least logically possible that all the priming in the attended conditions derived from concept or name priming. Pre-

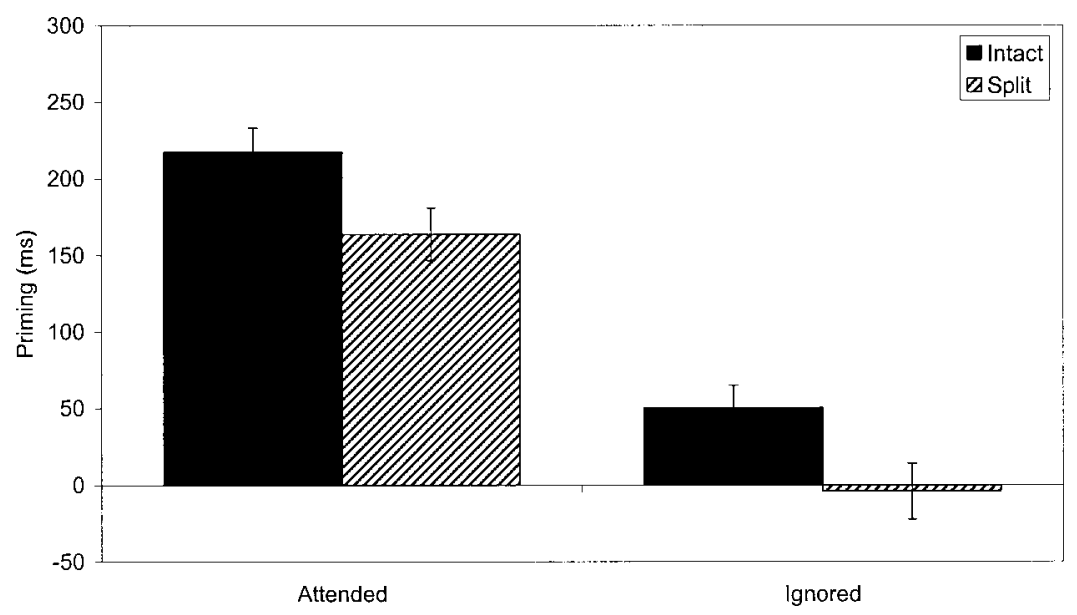

Figure 3. Priming means (baseline RT - RT in each experimental condition) for Experiment 1 as a function of whether the prime image was (a) attended or ignored and (b) intact or split $(N=42)$. Error bars represent standard errors. RT = response time. 
Table 1

Mean Response Times (RTs; in Milliseconds), Standard Errors, and Percentages of Error for Conditions in Experiment 1

\begin{tabular}{lrrrrrr}
\hline & \multicolumn{2}{c}{ Attended } & & \multicolumn{2}{c}{ Ignored } & \\
\cline { 2 - 3 } Variable & Intact & Split & & Intact & Split & Unprimed \\
\hline RT & 595 & 649 & & 762 & 816 & 813 \\
$S E$ & 19 & 15 & & 15 & 19 & 17 \\
\% error & 7 & 15 & & 11 & 13 & 14 \\
\hline
\end{tabular}

vious research (Biederman \& Cooper, 1991a, 1991b, 1992; Biederman \& Gerhardstein, 1993; Stankiewicz et al., 1998) has shown that priming from attended images is largely (although not completely) visual. However, this fact does not imply that the priming observed in the current paradigm is necessarily visual. As such, it is necessary to tease apart what fraction of the priming observed in the current paradigm is visual and what fraction nonvisual (e.g., concept or name priming).

Experiment 2 was designed to estimate what fraction of the priming observed in Experiment 1 was due to visual (as opposed to concept and/or name) priming. To this end, images in the identical-image conditions of Experiment 1 (attended-intact, ignored-intact) were replaced with images of objects having the same basic-level name as the corresponding probe object but a different shape (same-name-different-exemplar [SNDE], following Biederman \& Cooper, 1991a, 1991b, 1992; Biederman \& Gerhardstein, 1993; Stankiewicz et al., 1998). For example, if a grand piano (basic-level name piano) served as a probe object, then Experiment 2 presented an intact image of an upright piano ("piano") in the SNDE prime condition (instead of an identical grand piano) and a split version of the image of the grand piano in the split conditions. The conditions with split images (attendedsplit, ignored-split) were identical to those in Experiment 1 in that the split object of the prime display was followed by an intact probe object of the same exemplar. If any of the priming observed for attended objects in the split condition in Experiment 2 is specifically visual, then we expect more priming with split image (grand piano) primes than with SNDE (upright piano) primes. By contrast, if all the priming observed with split images in Experiment 1 was simply name or concept priming, then the SNDE images should prime as much as (or more than) the split images (see Biederman \& Cooper, 1991a; Stankiewicz et al., 1998).

\section{Method}

Participants. Forty-two native English speakers with normal or corrected-to-normal vision participated for credit in introductory psychology courses at the University of California, Los Angeles, and at Goldsmiths College, University of London, London, United Kingdom.

Materials. The experiment used a set of 84 objects in 42 SNDE pairs. Half were taken from the Snodgrass and Vanderwart (1980) set used in Experiment 1, and the corresponding SNDE exemplars were line drawings of similar style.

Procedure. The conditions in Experiment 2 were identical to those of Experiment 1 except that the intact primes were replaced by SNDE primes. Thus, each probe image in the intact conditions was paired with an intact probe image of a different object with the same name (rather than being paired with itself, as in Experiment 1). As in Experiment 1, all objects appeared equally often in each of the seven prime-probe conditions.

\section{Results and Discussion}

Trials in which either the prime or probe were named incorrectly were excluded (20.1\%), as were voice-key errors (3.7\%). Figure 4 and Table 2 show the priming results in each condition. A 2 (attention: attended vs. ignored) $\times 2$ (prime type: SNDE vs. split image) $\times 2$ (unprobed prime: intact vs. split) within-subject ANOVA revealed reliable main effects of attention, $F(1,41)=$ $34.53, p<.001$, and prime type, $F(1,41)=6.10, p<.02$. The Attention $\times$ Prime Type interaction was also reliable, $F(1,41)=$ $10.86, p<.002$. A Newman-Keuls post hoc analysis on the interaction revealed that the difference between the attendedSNDE and attended-split conditions was statistically reliable $(p<$ $.008)$, but the difference between the ignored-SNDE and ignoredsplit conditions was not $(p>.60)$. There was no main effect of

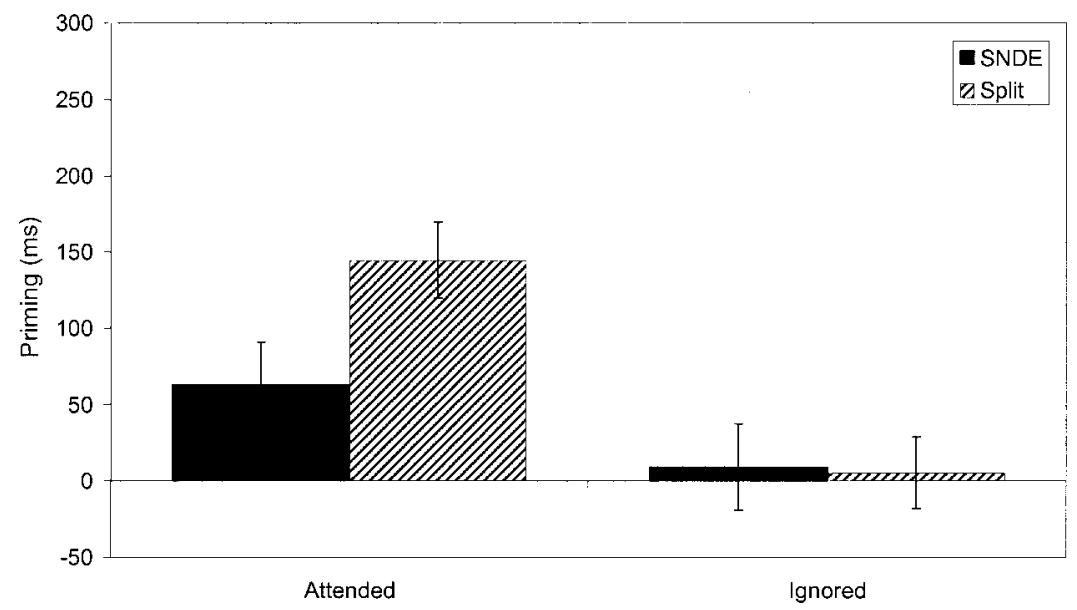

Figure 4. Priming means (baseline RT - RT in each experimental condition) for Experiment 2 as a function of whether the prime object was (a) attended or ignored and (b) a same-name-different-exemplar (SNDE) or a split image $(N=42)$. Error bars represent standard errors. RT $=$ response time. 
Table 2

Mean Response Times (RTs; in Milliseconds), Standard Errors, and Percentages of Error for Conditions in Experiment 2

\begin{tabular}{lccrrrr}
\hline & \multicolumn{2}{c}{ Attended } & & \multicolumn{2}{c}{ Ignored } & \\
\cline { 2 - 3 } Variable & SNDE & Split & & SNDE & Split & Unprimed \\
\hline RT & 689 & 615 & & 735 & 748 & 749 \\
$S E$ & 22 & 19 & & 24 & 25 & 16 \\
\% error & 18 & 24 & & 22 & 14 & 22 \\
\hline
\end{tabular}

Note. $\quad \mathrm{SNDE}=$ same-name-different-exemplar.

unprobed prime, and there were no interactions involving that factor ( $p s>.14$ for all comparisons). There were no indications of a speed-accuracy trade-off.

Analysis of each priming condition was carried out to determine which type of prime display caused savings in RT for the probe display (i.e., faster naming responses relative to unprimed probes). Priming was reliably greater (Bonferroni corrected) than zero in the attended-split condition, $t(41)=5.89, p<.001$, was almost so in the attended-SNDE condition, $t(41)=2.27, p<.03$ (critical value: $p<.0125)$, but was not in either the ignored-SNDE, $t(41)<1$, or the ignored-split condition, $t(41)<1$. Thus, SNDE and split images primed the corresponding probe image when attended but not when ignored.

Experiment 2 showed that an intact probe was primed more by an attended split image than by an intact different exemplar of the same basic-level category. The priming advantage for attended split objects over attended intact SNDEs was about $80 \mathrm{~ms}$. Because in both cases participants responded with the same name in prime and probe trials, this difference indicates a strong visual component to the priming in the attended and ignored conditions. Indeed, the lack of any priming for unattended SNDE primes suggests that all the priming observed in the unattended condition of Experiment 1 was specifically visual.

Experiment 2 found no priming in either of the ignored conditions. It therefore not only replicated the lack of priming for ignored split images in Experiment 1 but also produced no priming that was reliably greater than zero for SNDE prime images (replicating Stankiewicz et al., 1998). These last results contrast with the findings of Tipper (1985), who showed evidence of negative semantic priming for ignored stimuli (see Stankiewicz et al., 1998, for a discussion).

\section{Experiment 3}

Experiments 1 and 2 together demonstrated visual priming for intact and split images in the attended conditions, but only intact images were primed in the ignored conditions. This pattern of priming is expected on the account that the visual system generates holistic representations of ignored images and analytic representations of attended images (Hummel, 2001; Hummel \& Stankiewicz, 1996a). However, an alternative interpretation of the results of Experiments 1 and 2 is that all the observed priming resides in early visual representations (i.e., rather than in the representations responsible for object recognition, as assumed by the hybrid model) and that identical images simply prime one another more than nonidentical images, and attended images prime one another more than unattended images. If this "early-priming" account is correct, then the advantage for identical images over nonidentical images and the advantage for attended images over unattended images could combine to yield the effects observed in Experiment 1. Although this interpretation is challenged by the results of Stankiewicz and Hummel (2002), which showed that priming for ignored images is invariant with translation and scale - and by the fact that intact and split versions of the same image present corresponding features to very different retinal locations and, therefore, probably activate few of the same neurons in early visual processing at areas V1 and V2 - it cannot be ruled out completely only on the basis of the results of Experiments 1 and 2.

Experiment 3 was designed to establish whether the results of Experiments 1 and 2 reflect simply priming in early visual representations or a reliance on holistic processing for ignored images, as predicted by the hybrid model. The logic of Experiment 3 is based on an assumption about the locus of the visual priming observed in these and other experiments. Priming is a form of learning, leading Cooper, Biederman, and Hummel (1992) to speculate that one likely locus of visual priming is the point at which visual representations of object shape make contact with representations stored in LTM (see Biederman \& Cooper, 1991a, for evidence supporting this conjecture). Thus, visual priming-including priming of ignored images - must reflect the activation of preexisting representations in LTM. Indeed, Potter (1976) has shown that failing to attend to a stimulus results in a failure to encode it into LTM. If an image does not have an existing representation in LTM, then ignoring that image on one occasion should not prime recognition of the very same image on a subsequent occasion. Consistent with this reasoning, Stankiewicz (1997) showed that ignoring an upside-down image on one trial does not prime recognition of the very same (i.e., upside-down) image on the next trial.

Applied to our current paradigm, the logic is as follows: If the results of Experiments 1 and 2 reflect the role of holistic representations in the recognition of ignored images, and if these holistic representations are encoded in LTM in an intact (rather than a split) format, then ignoring a split image on one occasion should not prime recognition of the very same image on a subsequent occasion. However, if the results of Experiments 1 and 2 reflect priming of early visual features (in both the attended and ignored cases), then ignoring a split image on one trial should prime recognition of that image on the subsequent trial. By contrast, both accounts would predict that attending to a split image on one trial should permit the encoding and, therefore, priming of that image.

\section{Method}

Participants. Thirty-six English speakers with normal or corrected-tonormal vision participated for money or for credit in introductory psychology courses at Goldsmiths College, University of London.

Materials. The experiment used a set of 84 objects; 36 were used in prime-probe target pairs, and the rest were fillers for attended and ignored primes. The items were taken from the Snodgrass and Vanderwart (1980) set and were similar to those used in Experiment 1.

Procedure. Experiment 3 used the same procedure as Experiment 1, except that (a) half of the time, the probe was a split image (recall that in Experiment 1, all probe images were intact), and (b) the configuration of 


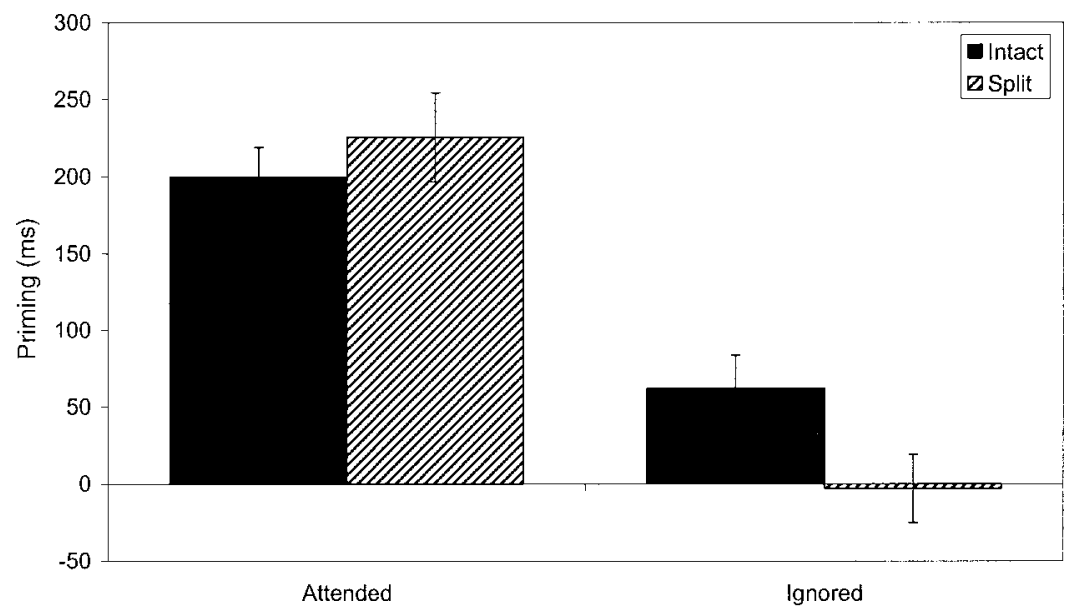

Figure 5. Priming means (baseline RT - RT in each experimental condition) for Experiment 3 as a function of whether (a) the prime object was attended or ignored and (b) both prime and probe were split or intact $(N=$ 36). Error bars represent standard errors. RT $=$ response time.

the probe image (split or intact) was always the same as the configuration (intact or split) of the corresponding prime. Naturally, this constraint does not apply to unprimed baseline images, which have no corresponding prime. Unprobed items for prime trials were always intact images, except for the unprimed-split (baseline) condition.

\section{Results and Discussion}

Trials in which either the prime or probe were named incorrectly were excluded (15.7\%), together with voice-key errors $(4.9 \%)$. Priming was calculated in the same way as in Experiment 1, except that two different baseline conditions (unprimed-split and unprimed-intact) were used to calculate the corresponding priming for split and for intact conditions. Figure 5 and Table 3 depict the amount of priming observed in each condition. A 2 (attention: attended vs. ignored) $\times 2$ (configuration: intact vs. split) withinsubject ANOVA revealed a reliable main effect of attention, $F(1$, $35)=119.42, p<.001$, but not configuration, $F(1,35)<1$. However, the Attention $\times$ Configuration interaction was reliable, $F(1,35)=12.54, p<.01$. A Newman-Keuls post hoc analysis of the interaction revealed a reliable difference between the ignoredintact and ignored-split conditions $(p<.002)$ but not between the attended-intact and attended-split conditions $(p>.17)$.

Analysis of each priming condition was carried out to determine which type of prime display caused savings in RT for the probe display (i.e., faster naming responses relative to unprimed probes). Priming was reliably greater than zero (Bonferroni corrected) in the attended-intact, $t(35)=7.67, p<.001$, attended-split, $t(35)=$ 11.93, $p<.001$, and ignored-intact conditions, $t(35)=2.81, p<$ .01 , but it was not reliably greater than zero in the ignored-split condition, $t(35)<1$. $^{1}$

Figure 5 shows that the pattern of priming for attended conditions differs somewhat from those of Experiments 1 and 2. When attended, a split image primed itself just as much as an intact image primed itself. At first blush, this result seems surprising, especially given the failure of ignored split images to prime themselves at all. However, there were both more errors and longer latencies for split images, suggesting that split images are simply harder to recognize (see Table 3), making it likely that they profit more from priming than do intact images (see, e.g., Potter, 1976; Rensink, 2000).

Because Experiment 3 differed from Experiments 1 and 2 in the use of different baselines, we also carried out analyses of the raw probe latencies. A 3 (prime type: attended, ignored, unprimed) $\times$ 2 (configuration: intact vs. split) ANOVA on probe latencies revealed main effects of prime type, $F(2,70)=94.71, p<.001$, and configuration, $F(1,35)=29.83, p<.001$, and a significant Prime Type $\times$ Configuration interaction, $F(2,70)=5.54, p<.01$. A Newman-Keuls post hoc analysis of the interaction revealed no difference between latencies for split images compared with those for intact images in the attended conditions $(p>.11)$ but reliable differences in the ignored conditions $(p<.001)$ and unprimed conditions $(p<.01)$. Thus, the pattern of results shown in Figure 5 is not simply the result of baseline differences in the recognition of split images.

A 3 (prime type: attended, ignored, unprimed) $\times 2$ (configuration: intact vs. split) ANOVA on error rates (excluding voice-key errors) revealed only a main effect of configuration, $F(1,35)=$ $12.87, p<.01$, with error rates for split images higher than for

\footnotetext{
${ }^{1}$ The size difference between the cuing square and the object was $0.5^{\circ}$ of visual angle. A reviewer suggested that this may be a small enough separation to allow completion of the split images. Thus, the difference between attended and ignored split-image conditions could have arisen from there being completion only in the former case. Although there are several reasons for discounting completion as an explanation of the pattern of results in Experiments 1-3, the most straightforward way was to assess the effect of a larger separation between the cuing square and image. In a replication, 36 participants took part in an identical experiment to Experiment 3 , except that the size difference between cuing square and image was now $1^{\circ}$ of visual angle. The statistical pattern of results was very similar; the only two reliable effects were for attention, $F(1,35)=84.44$, $p<.001$, and for the Attention $\times$ Configuration interaction, $F(1,35)=$ $10.47, p<.01$. Thus, there was reliable priming in all conditions (attended-intact: $160.6 \mathrm{~ms}$; attended-ignored: $204.3 \mathrm{~ms}$; ignored-intact: 49.9 $\mathrm{ms})$, except for the ignored-split condition $(16.8 \mathrm{~ms})$.
} 
Table 3

Mean Response Times (RTs; in Milliseconds), Standard Errors, and Percentages of Error for Conditions in Experiment 3

\begin{tabular}{lrrrrrrrr}
\hline & \multicolumn{2}{c}{ Attended } & & \multicolumn{2}{c}{ Ignored } & & \multicolumn{2}{c}{ Unprimed } \\
\cline { 2 - 3 } Variable & Intact & Split & & Intact & Split & & Intact & Split \\
\hline RT & 630 & 662 & & 768 & 890 & & 830 & 887 \\
$S E$ & 16 & 18 & & 19 & 27 & & 25 & 24 \\
\% error & 8 & 20 & & 17 & 17 & & 12 & 20 \\
\hline
\end{tabular}

intact images. Thus, there is no evidence of a speed-accuracy trade-off in any condition.

As predicted by the hybrid model - but not by the early visual priming hypothesis-Experiment 3 showed that split images primed themselves when they were attended but not when they were ignored, whereas intact images primed themselves whether they were attended or not. In addition, in the attended conditions, split images primed themselves just as much as intact images. Experiment 3 thus demonstrated that the lack of priming for ignored split images in Experiment 1 cannot be attributed to a general decrease of priming in response to split images and, more generally, that the priming pattern observed in Experiment 1 cannot be explained by a simple "more priming for identical images" (low-level visual) account.

\section{General Discussion}

The results of these three experiments strongly support the central theoretical tenet of the hybrid model of object recognition (Hummel, 2001; Hummel \& Stankiewicz, 1996a), that object recognition is based on a hybrid analytic/holistic representation of object shape. In Experiment 1, attended intact, attended split, and ignored intact images primed subsequent recognition of corresponding intact images, whereas ignored split images did not prime their intact counterparts. This pattern of effects is predicted by the hybrid account because attended images are represented both analytically and holistically, whereas ignored images are represented only holistically. Since a holistic representation of a split image has little or nothing in common with a holistic representation of its intact counterpart, ignoring a split image (and thereby representing it only holistically) is not expected to prime its intact counterpart. Ignoring an intact image, by contrast, is expected to prime its intact counterpart. Priming is expected for an analytic representation of a split image because it has a great deal in common with an analytic representation of its intact counterpart; thus, attending to a split image is expected to prime its intact counterpart.

Experiment 1 also revealed another important aspect of the hybrid account. Because an attended image is expected to be represented both analytically and holistically, attending to an intact image (thereby priming both the analytic and holistic representations) is expected to prime that image more than attending to its split counterpart (which is expected to prime the analytic representation only). The advantage for an intact prime (relative to a split prime) was exactly the same $(\sim 50 \mathrm{~ms})$ in both the attended and the ignored conditions. This additive effect of configuration with attention (like the additive effects of view [identical vs. reflected] and attention observed by Stankiewicz et al., 1998) suggests that the analytic and holistic representations are processed in parallel and make independent contact with object memory (see also Stankiewicz et al., 1998).

Experiment 2 demonstrated that a substantial fraction (at least $80 \mathrm{~ms}$ ) of the observed priming for attended objects was specifically visual (rather than simply name or concept priming). Experiment 3 showed that ignored split images did not prime even themselves; but, when attended, split images primed themselves at least as much as intact images primed themselves. These results indicate that the results of Experiments 1 and 2 reflect visual priming at the interface of shape perception and object memory and cannot be accounted for by simply assuming that identical images always prime themselves more than nonidentical images and that attended images always prime themselves more than unattended images. Together, the results of Experiments 1-3 strongly suggest that, as predicted by the hybrid model, the visual system represents attended images both analytically and holistically and represents ignored images only holistically.

One of the most striking aspects of the findings presented here is that the results of Experiment 1 (Figure 3) are nearly an exact numerical replication of the findings of Stankiewicz et al. (1998, Experiment 1) who used left-right reflections rather than split images. In both studies, identical images enjoyed a priming advantage of approximately $50 \mathrm{~ms}$ over nonidentical (i.e., split or reflected) images in both attended and ignored conditions. The fact that configural distortions (splitting an image) and left-right reflection have such similar effects on attended and unattended images suggests that similar mechanisms are at work in both cases (as predicted by the hybrid model). Namely, attention permits the visual system to generate an analytic representation robust to both configural distortions and left-right reflection, whereas in the absence of attention, the visual system must rely on holistic representations that are robust to neither of these manipulations.

The findings reported here are inconsistent with the majority of models of object recognition currently in the literature. Viewbased models (e.g., Poggio \& Edelman, 1990; Tarr \& Bülthoff, 1995; Ullman \& Basri, 1991) and their relatives (e.g., Edelman \& Intrator, 2000, 2003; Riesenhuber \& Poggio, 1999) are based strictly on varieties of holistic coding and cannot account for the fact that a split image can visually prime its intact counterpart. More generally, these models are inconsistent with the role of relations in shape perception (Hummel, 2000; Hummel \& Biederman, 1992) and with the capacity of the human visual system to represent aspects of object shape independently of one another (i.e., nonholistically; Stankiewicz, 2002). Pure structural description theories (e.g., Biederman, 1987; Hummel \& Biederman, 1992; Marr \& Nishihara, 1978), as well as feature-listing theories (Mel, 1997; Mel \& Fiser, 2000), provide a natural account of the fact that split images can visually prime their intact counterparts, but these models fail to account for the role of visual attention in this capacity. Feature-based models, in particular, predict that visual priming should persist over many kinds of configural distortions (including random scrambling of an object's features about the image; Hummel \& Biederman, 1992; Mel, 1997), regardless of whether the distorted image is attended or ignored. Accounting both for the fact that split images can prime their intact counterparts and for the fact that this priming depends on visual attention requires a hybrid account in which attention is required to generate 
a splitting-robust representation of shape. Hybrid accounts based on fragments derived from learned views (e.g., Edelman \& Intrator, 2000, 2003) do not predict visual priming from split images to their intact counterparts because the proposed fragments are tied to specific locations in the image. Moreover, fragment theory predicts that multiple fixations are needed during initial encoding in order to establish the various location-dependent object fragments (Edelman \& Intrator, 2003). In our experiments split images primed subsequent intact probe images even though their presentation duration was too short to permit saccades.

The findings presented here have additional important implications for theories of object recognition in general and for the hybrid model in particular. The findings strongly suggest that the analytic and holistic representations work in parallel rather than in a serial manner (as predicted by the hybrid model). It is not simply a matter of "early" priming for ignored images and "early and late" priming for attended images. A serial model of this kind could, in principle, account for the results of our Experiments 1 and 2 and for the effects reported by Stankiewicz et al. (1998), who showed that a change in viewpoint (left-right reflection) was associated with a reduction in priming for both attended and ignored objects. However, such an account cannot explain the priming effects observed in Experiment 3, in which split images did not even prime themselves when ignored.

Our findings also have implications for the breakdown of object recognition with brain damage. Davidoff and Warrington (1999, 2001) reported case studies of patients who were extremely poor at recognizing object parts or "exploded" objects, even though they could name intact objects (in familiar views). In terms of the hybrid model, it would seem that these patients' holistic route was unimpaired, allowing them to recognize objects presented in intact familiar views, whereas their analytic route seemed to be impaired, because recognition of parts was poor.

In conclusion, the experiments reported here demonstrated that the visual representation generated in response to an attended image is qualitatively different from that generated in response to an ignored image. Although recognition takes place in both cases, it is mediated by an analytic representation when the image is attended and by a holistic representation when the image is ignored. These findings suggest a very specific answer to the question raised at the beginning of this article: How can the visual representation of shape have some properties that demand explanation in terms of analytic representations and simultaneously have other properties that are strictly inconsistent with analytic representations? It appears that the visual system represents shape analytically when it can but represents it holistically when it must.

\section{References}

Biederman, I. (1987). Recognition-by-components: A theory of human image understanding. Psychological Review, 94, 115-147.

Biederman, I., \& Cooper, E. E. (1991a). Evidence for complete translational and reflectional invariance in visual object priming. Perception, 20, 585-593.

Biederman, I., \& Cooper, E. E. (1991b). Priming contour-deleted images: Evidence for intermediate representations in visual object recognition. Cognitive Psychology, 23, 393-419.

Biederman, I., \& Cooper, E. E. (1992). Size invariance in visual object priming. Journal of Experimental Psychology: Human Perception and Performance, 18, 121-133.
Biederman, I., \& Gerhardstein, P. C. (1993). Recognizing depth-rotated objects: Evidence and conditions for three-dimensional viewpoint invariance. Journal of Experimental Psychology: Human Perception and Performance, 19, 1162-1182.

Biederman, I., \& Gerhardstein, P. C. (1995). Viewpoint-dependent mechanisms in visual object recognition: Reply to Tarr and Bülthoff (1995). Journal of Experimental Psychology: Human Perception and Performance, 21, 1506-1514.

Cave, C. B., \& Kosslyn, S. M. (1993). The role of parts and spatial relations in object identification. Perception, 22, 229-248.

Cooper, E. E., Biederman, I., \& Hummel, J. E. (1992). Metric invariance in object recognition: A review and further evidence. Canadian Journal of Psychology, 46, 191-214.

Davidoff, J., \& Warrington, E. K. (1999). The bare bones of object recognition: Implications from a case of object recognition impairment. Neuropsychologia, 37, 279-292.

Davidoff, J., \& Warrington, E. K. (2001). A particular difficulty in discriminating between mirror images. Neuropsychologia, 39, 1022-1036.

Edelman, S. (1998). Representation is representation of similarities. Behavioral and Brain Sciences, 21, 449-498.

Edelman, S., \& Intrator, N. (2000). (Coarse coding of shape fragments) + (retinotopy) $\approx$ representation of structure. Spatial Vision, 13, 255-264.

Edelman, S., \& Intrator, N. (2003). Towards structural systematicity in distributed, statically bound visual representations. Cognitive Science, 27, 73-110.

Hummel, J. E. (2000). Where view-based theories break down: The role of structure in shape perception and object recognition. In E. Dietrich \& A. Markman (Eds.), Cognitive dynamics: Conceptual change in humans and machines (pp. 157-185). Mahwah, NJ: Erlbaum.

Hummel, J. E. (2001). Complementary solutions to the binding problem in vision: Implications for shape perception and object recognition. Visual Cognition, 8, 489-517.

Hummel, J. E. (2003a). The complementary properties of holistic and analytic representations of object shape. In M. Peterson \& G. Rhodes (Eds.), Perception of faces, objects, and scenes: Analytic and holistic processes (pp. 212-234). New York: Oxford University Press.

Hummel, J. E. (2003b). "Effective systematicity" in, "effective systematicity" out: A reply to Edelman and Intrator (2003). Cognitive Science, $27,327-329$.

Hummel, J. E., \& Biederman, I. (1992). Dynamic binding in a neural network for shape recognition. Psychological Review, 99, 480-517.

Hummel, J. E., \& Stankiewicz, B. J. (1996a). An architecture for rapid, hierarchical structural description. In T. Inui \& J. McClelland (Eds.), Attention and performance XVI: Information integration in perception and communication (pp. 93-121). Cambridge, MA: MIT Press.

Hummel, J. E., \& Stankiewicz, B. J. (1996b). Categorical relations in shape perception. Spatial Vision, 10, 201-236.

Hummel, J. E., \& Stankiewicz, B. J. (1998). Two roles for attention in shape perception: A structural description model of visual scrutiny. Visual Cognition, 5, 49-79.

Intraub, H. (1981). Identification and processing of briefly glimpsed visual scenes. In D. Fisher, R. A. Monty, \& J. W. Sender (Eds.), Eye movements: Cognition and visual perception (pp. 181-190). Hillsdale, NJ: Erlbaum.

Jolicœur, P. (1985). The time to name disoriented natural objects. Memory \& Cognition, 13, 289-303.

Lawson, R. (1999). Achieving visual object constancy across plane rotation and depth rotation. Acta Psychologica, 102, 221-245.

Logan, G. D. (1994). Spatial attention and the apprehension of spatial relations. Journal of Experimental Psychology: Human Perception and Performance, 20, 1015-1036.

Lowe, D. G. (1987). The viewpoint consistency constraint. International Journal of Computer Vision, 1, 57-72. 
Luck, S. J., \& Vogel, E. K. (1997, November 20). The capacity of visual working memory for features and conjunctions. Nature, 390, 279-281.

Marr, D., \& Nishihara, H. K. (1978). Representation and recognition of the spatial organization of three-dimensional shapes. Proceedings of the Royal Society of London, Series B, 200, 269-294.

Mel, B. W. (1997). SEEMORE: Combining color, shape, and texture histogramming in a neurally inspired approach to visual object recognition. Neural Computation, 9, 777-804.

Mel, B. W., \& Fiser, J. (2000). Minimizing binding errors using learned conjunctive features. Neural Computation, 12, 247-278.

Murray, J. E. (1995). Negative priming by rotated objects. Psychonomic Bulletin \& Review, 2, 534-537.

Oram, M. W., \& Perrett, D. I. (1992). The time course of neural responses discriminating different views of the face and head. Journal of Neurophysiology, 68, 70-84.

Palmer, S. E. (1977). Hierarchical structure in perceptual representation. Cognitive Psychology, 9, 441-474.

Palmer, S. E. (1978). Structural aspects of similarity. Memory \& Cognition, 6, 91-97.

Poggio, T., \& Edelman, S. (1990, January 18). A neural network that learns to recognize three-dimensional objects. Nature, 343, 263-266.

Potter, M. C. (1976). Short-term conceptual memory for pictures. Journal of Experimental Psychology: Human Learning and Memory, 2, 509522 .

Rensink, R. A. (2000). The dynamic representation of scenes. Visual Cognition, 7, 17-42.

Riesenhuber, M., \& Poggio, T. (1999). Hierarchical models of object recognition in cortex. Nature Neuroscience, 2, 1019-1025.

Saiki, J., \& Hummel, J. E. (1996). Attribute conjunctions and the part configuration advantage in object category learning. Journal of Experimental Psychology: Learning, Memory and Cognition, 22, 1002-1019.

Saiki, J., \& Hummel, J. E. (1998a). Connectedness and the integration of parts with relations in shape perception. Journal of Experimental Psychology: Human Perception and Performance, 24, 227-251.

Saiki, J., \& Hummel, J. E. (1998b). Connectedness and part-relation integration in shape category learning. Memory \& Cognition, 26, 1138 1156.

Snodgrass, J. G., \& Vanderwart, M. (1980). A standardized set of 260 pictures: Norms for name agreement, image agreement, familiarity, and visual complexity. Journal of Experimental Psychology: Human Learning and Memory, 6, 174-215.

Stankiewicz, B. J. (1997). The role of attention in viewpoint-invariant object recognition. Unpublished doctoral dissertation, University of California, Los Angeles.

Stankiewicz, B. J. (2002). Empirical evidence for independent dimensions in the visual representation of three-dimensional shape. Journal of
Experimental Psychology: Human Perception and Performance, 28, 913-932.

Stankiewicz, B. J., \& Hummel, J. E. (2002). Automatic priming for translation- and scale-invariant representations of object shape. Visual Cognition, 9, 719-739.

Stankiewicz, B. J., Hummel, J. E., \& Cooper, E. E. (1998). The role of attention in priming for left-right reflections of object images: Evidence for a dual representation of object shape. Journal of Experimental Psychology: Human Perception and Performance, 24, 732-744.

Tarr, M. J., \& Bülthoff, H. H. (1995). Is human object recognition better described by geon structural descriptions or by multiple views? Comment on Biederman and Gerhardstein (1993). Journal of Experimental Psychology: Human Perception and Performance, 21, 1494-1505.

Tarr, M. J. \& Pinker, S. (1989). Mental rotation and orientation dependence in shape recognition. Cognitive Psychology, 21, 233-283.

Tarr, M. J., \& Pinker, S. (1990). When does human object recognition use a viewer-centered reference frame? Psychological Science, 1, 253-256.

Tipper, S. P. (1985). The negative priming effect: Inhibitory effects of ignored primes. Quarterly Journal of Experimental Psychology: Human Experimental Psychology, 37(A), 571-590.

Tipper, S. P., \& Driver, J. (1988). Negative priming between pictures and words in a selective attention task: Evidence for semantic processing of ignored stimuli. Memory \& Cognition, 16, 64-70.

Treisman, A., \& DeSchepper, B. (1996). Object tokens, attention, and visual memory. In T. Inui \& J. McClelland (Eds.), Attention and performance XVI: Information integration in perception and communication (pp. 15-46). Cambridge, MA: MIT Press.

Treisman, A., \& Gelade G. (1980). A feature integration theory of attention. Cognitive Psychology, 12, 97-136.

Tversky, B., \& Hemenway, K. (1984). Objects, parts, and categories. Journal of Experimental Psychology: General, 113, 169-193.

Ullman, S. (1989). Aligning pictorial descriptions: An approach to object recognition. Cognition, 32, 193-254.

Ullman, S. (1996). High-level vision: Object recognition and visual cognition. Cambridge MA: MIT Press.

Ullman, S., \& Basri, R. (1991). Recognition by linear combinations. IEEE Transactions on Pattern Analysis and Machine Intelligence, 13, 9921006.

Winston, P. (1975). Learning structural descriptions from examples. In P. Winston (Ed.), The psychology of computer vision (pp. 157-209). New York: McGraw-Hill.

Received July 23, 2002

Revision received March 21, 2003

Accepted July 16, 2003

\section{Wanted: Your Old Issues!}

As APA continues its efforts to digitize journal issues for the PsycARTICLES database, we are finding that older issues are increasingly unavailable in our inventory. We are turning to our long-time subscribers for assistance. If you would like to donate any back issues toward this effort (preceding 1982), please get in touch with us at journals@apa.org and specify the journal titles, volumes, and issue numbers that you would like us to take off your hands. 